Case Report

\title{
Preoperative Transcatheter Arterial Embolization and En-Bloc Resection for Giant Non-Functioning Left Adrenocortical Carcinoma: A Case Report
}

\author{
Giorgio Lucandri $^{I^{*}}$, Giuseppe Mezzetti ${ }^{1}$, Marco Maria Lirici ${ }^{1}$, Alessia Fassari ${ }^{1}$, Vito Pende ${ }^{1}$, Simone Vagnarelli ${ }^{2}$, \\ Daniela Bosco $^{3}$, Antonio Spada ${ }^{3}$, Assunta Santonati ${ }^{3}$, Domenico Campagna ${ }^{4}$ and Massimo Farina ${ }^{1}$ \\ ${ }^{1} 1^{\text {st }}$ Department of Surgery, San Giovanni-Addolorata Hospital, Rome, Italy \\ ${ }^{2}$ Department of Radiology, San Giovanni-Addolorata Hospital, Rome, Italy \\ ${ }^{3}$ Departmental Unit of Endocrinology, San Giovanni-Addolorata Hospital, Rome, Italy \\ ${ }^{4}$ Department of Pathology, San Giovanni-Addolorata Hospital, Rome, Italy

\section{A R T ICLE INFO} \\ Article history: \\ Received: 8 June, 2021 \\ Accepted: 21 June, 2021 \\ Published: 8 July, 2021 \\ Keywords: \\ Adrenocortical carcinoma \\ mitotane \\ multi-visceral resection \\ non-secretory tumor \\ retroperitoneal tumor \\ transcatheter arterial embolization

\begin{abstract}
A B S T R A C T
Objective: To demonstrate efficacy and relative safety of compartment surgery in a case of large sized adrenocortical carcinoma (ACC), whenever associated with preoperative transcatheter arterial embolization (TAE) and adjuvant treatment with mitotane.

Introduction: ACC is a rare illness; non-functioning lesions account for 40-55\% of patients, may reach a large size, and show a clear attitude to infiltrate neighbouring organs.

Case Report: Middle aged male visited for a huge left abdominal mass; contrast CT scan showed origin from the left adrenal lodge and possible extension of the tumor to surrounding structures. Preoperative adrenal work-up confirmed non-functioning nature of the adrenal mass. In order to allow a safe compartment excision and to decrease blood loss, preoperative angiography and TAE was performed. Patient underwent en-bloc resection of the mass, together with nephrectomy and distal splenopancreasectomy. No postoperative transfusions were required. Patient received adjuvant therapy with mitotane for 36 months since surgery, without any sign of tumor recurrence.

Conclusion: An aggressive surgical approach seems justified also in locally advanced and infiltrating ACC; compartment resection is mandatory in order to obtain adequate resection margins. Preoperative TAE decreases intraoperative blood loss and need for transfusions. Adjuvant treatment with mitotane may
\end{abstract} \\ contribute to reach remarkable disease-free survival.
}

\section{Introduction}

Carcinoma of the adrenal gland or suprarenal carcinoma (ACC) is a rare illness and Literature accounts only for case reports and small series. It represents $0.2 \%$ of the causes of death from cancer, with prevalence for females (male/female ratio 1:2.5) and higher incidence during $4-5^{\text {th }}$ decade of life (median age at diagnosis 47 years) [1]. ACC can be functionally active in $60 \%$ of patients; in these cases, illness can be clinically detected by an excess of hormonal secretion, and a complete adrenal functional study usually leads to prompt diagnosis [2]. The remaining $40 \%$ of patients develops nonfunctioning adrenal carcinoma; it usually grows asymptomatically until it reaches a great size and may infiltrate surrounding structures with consistent mass effect. This cancer attitude and delayed diagnosis may worsen patient's prognosis and lead to a consistent rate of distant metastasis and unresectable lesions [3].

Whenever feasible, also an aggressive approach with resection of additional surrounding organs seems justified: the rate of multivisceral exeresis in extended ACC ranges from 38 to 63\%; the most common organs removed result kidney (50-60\%), left liver (30-35\%), spleen (20$25 \%)$ and pancreatic tail $(10-15 \%)[4,5]$. Patients treated with radical extended adrenalectomy are related to a 5-year overall survival rate

${ }^{*}$ Correspondence to: Giorgio Lucandri, M.D., Ph.D., ${ }^{\text {st }}$ Department of Surgery, San Giovanni-Addolorata Hospital, Via dell'Amba Aradam 8, 00184, Rome, Italy; Tel: +393398472308; Fax: +390677055259; E-mail: glucandri@hsangiovanni.roma.it 
ranging from $16 \%$ to $44 \%$; the Weiss System malignancy criteria and other indicators such as status of resection margins, lymph node involvement, distant metastases, expression of Ki67 and age less than 40 years are related to patient's outcome and some of them have to be proven as independent prognostic factors at multivariate analysis [6-9].

Consistent size of the ACC, presence of compression phenomena, invasion of surrounding tissues and frequent need for para-aortic dissection may lead to a consistent intra-operative blood loss and necessity for repeated blood transfusions [5]. Preoperative Transcatheter Arterial Embolization (TAE) may be useful in reducing blood supply to the ACC, by injecting low-viscosity hydrophobic liquid in the feeding vessels; several experiences have been reported in recent Literature with clear benefit in reducing blood loss, need for transfusions and postoperative morbidity $[3,10,11]$. Patients with large nonfunctioning ACC and delayed diagnosis may benefit from preoperative TAE and aggressive surgical multi-visceral resection; addition with adjuvant therapy with mitotane may result in consistent disease-free survival, as in the case of a huge left ACC we observed and successfully treated.

\section{Case Presentation}

A 57-year-old Caucasian male visited at our outpatient endocrinological service in April 2018 for a 3-months history of moderate to severe left flank pain, associated to a $5 \mathrm{~kg}$ weight loss and increasing weakness; no history of lower urinary tract symptoms or gross hematuria was referred. The patient suffered from type 2 diabetes, treated with metformin 500 mg twice a day; his remaining medical and surgical history was not significant, and he did not consume other regular medications. At abdominal exploration, a hard palpable mass was felt in the left hypochondrium and mesogastrium, while the rest of the clinical examination revealed normal findings; in particular, neither symptoms nor signs of possible adrenal dysfunction were detected, and blood pressure was within range values.

A consultant surgeon hypothesized origin of the mass from left kidney or left adrenal gland and addressed patient to further investigations. Head and chest contrast $\mathrm{CT}$ scan revealed normal findings. Abdominal CT scan showed a well-defined extensive mass, of regular contours and with heterogeneous signs, suggesting hemorrhagic and necrotic areas, arising from left adrenal lodge. It measured $18 \times 11 \times 10 \mathrm{~cm}$ in its greatest dimensions. The mass crossed the midline and displaced the spleen, the pancreas, and the left kidney. On arterial phase, splenic artery was grossly displaced and stretched upwards (Figure 1A). Venous and delayed phase confirmed dis-homogeneous aspect of the tumor and respectively clear evidence of displacement and absence of cleavage with the left kidney (Figure 1B) and strict adhesion with lower margin of pancreatic body and tail (Figure 1C). Neither infiltration of the IVC nor endovascular thrombotic figures could be demonstrated; no enlarged lymph nodes could be detected.

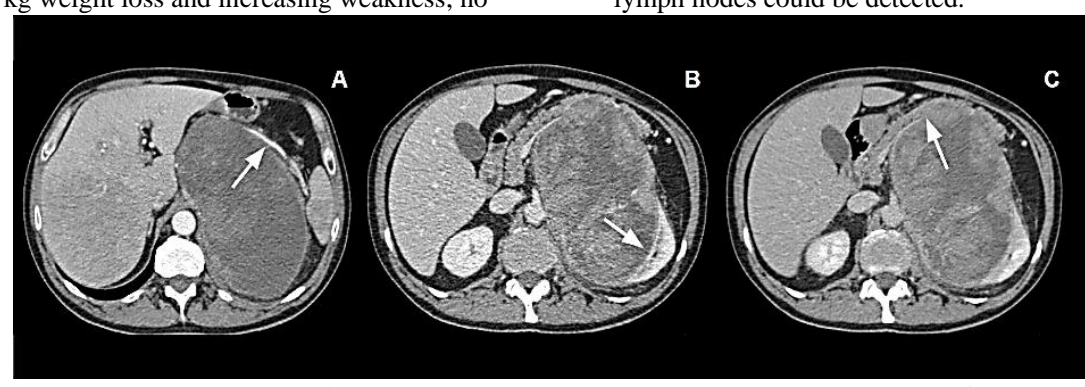

Figure 1: A) Arterial phase: Giant mass occupying left abdomen. Splenic artery appears stretched upward. B) Venous phase: Inhomogeneous mass displacing and probably infiltrating left kidney. C) Delayed phase: Absence of clear cleavage with pancreatic body and tail.

As adrenal origin of the tumor was confirmed, a functional adrenal workup was planned: it included measurement of serum aldosterone, potassium, renin, adrenocorticotrophic hormone levels, dexamethasone suppression test and 24-hour urinary metanephrine levels; all results were within ranges. Multidisciplinary oncologic board authorized a direct surgical approach, without further investigation or neoadjuvant therapies. Patient scored ASA 2 at anaesthesiological preoperative assessment; Hb level was $14.6 \mathrm{~g} / \mathrm{dl}$. Volume of the mass and its vascular supply made a postoperative ICU stay advisable.
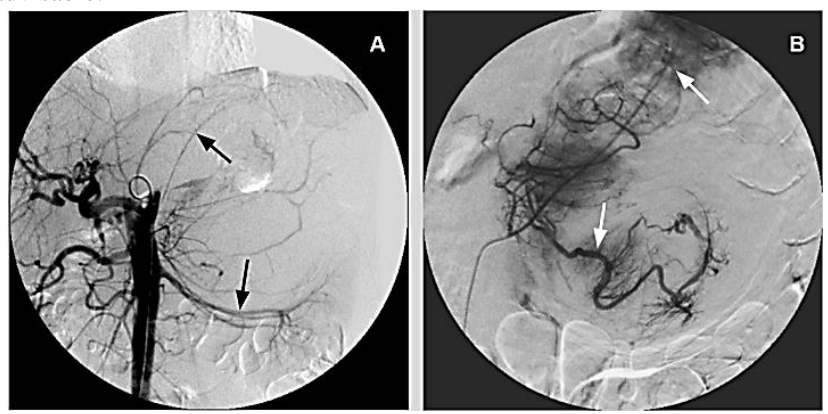

Figure 2: A) Preoperative Angiography: Evidence for stretched left renal artery and several pericapsular vessels. B) Post TAE control: Adequate distribution of Phil ${ }^{\circledR} 35 \%$ into feeding vessels.
Considering size of the mass and estimated intraoperative blood loss, patient underwent preoperative abdominal angiography (Figure 2A) and subsequent transcatheter arterial embolization (TAE). The largest feeding arterial vessels, coming from adrenal and polar artery were identified and embolized by using low viscosity Precipitating Hydrophobic Injectable Liquid (Phil ${ }^{\circledR}$ ) 35\% agent; post TAE control enhanced optimal distribution of the embolizing agent (Figure 2B). 
Surgery was performed with the patient lying on supine position and through a complete midline incision. Bookwalter ${ }^{\circledR}$ retractor was used to ensure adequate exposure of the left hypochondrium. After medial reflection of the descending colon and complete lowering of the splenic flexure, a huge left adrenal mass was found to displace the stomach, the spleen, and the left liver lobe and to clearly infiltrate the upper pole and the hilum of the left kidney; moreover, sharp, and smooth dissection of the root of the left transverse mesocolon and of the inferior aspect on the body and the tail of the pancreas confirmed absence of a surgical defined plane. Dissection of the huge adrenal mass resulted in parcellar but multiple discontinuations of splenic capsule. En-bloc resection of left adrenal mass together with radical nephrectomy and distal splenopancreasectomy was performed. Pancreatic stump was closed by using a $60 \mathrm{~mm}$. vascular stapler. The estimated intraoperative blood loss was $450 \mathrm{ml}$., and no perioperative blood transfusion was required. The postoperative course was uneventful; after 2 days ICU stay, patient was transferred to our ward. Both drains were removed on postoperative day 3 after laboratory reports confirmed the absence of amylase and lipase in the output. Bowel movement started on postoperative day 4 and the patient was discharged on postoperative day 9 .
Histological examination showed a capsulated adrenal specimen measuring $24 \times 22 \times 12 \mathrm{~cm}$ in its diameters and 2,080 g in weight. It was found in continuity with body and tail of the pancreas, which measured $\mathrm{cm} .10$ in its largest size, with the left kidney and proximal ureter; the spleen presented several disruptions on its capsule. After opening the specimen, adrenal mass was found highly necrotic, and the absence of cleavage both with kidney and pancreatic tissue was confirmed (Figure 3). According to Weiss criteria, tumor showed: presence of necrosis, high mitotic rate (> 5/50 HPF), high nuclear grade, clear cell component $<25 \%$, capsular involvement; immunohistochemistry revealed negative staining for CK7, CK20, CD31, pS100, CromogA and CKAE1/AE3 (Figure 4A) while synaptophysin, vimentin and MelanA were positive (Figure 4B) [9]. Ki67 index was 10-15\%. These findings supported diagnosis of adrenocortical carcinoma. In accordance with AJCC $8^{\text {th }} \mathrm{Ed}$. the tumor was staged as pT3 pNx R0. After evaluation at our outpatient oncological service, patient entered a protocol of treatment with mitotane (Lysodren ${ }^{\circledR}$ ) $3 \mathrm{gr} / \mathrm{die}$, reaching therapeutical blood levels of 14 $\mathrm{mg} / \mathrm{L}$ after 8 weeks and without developing significative side effects. Patient does not exhibit any clinical and radiological sign of recurrent or metastatic disease at 36 months since surgical treatment.

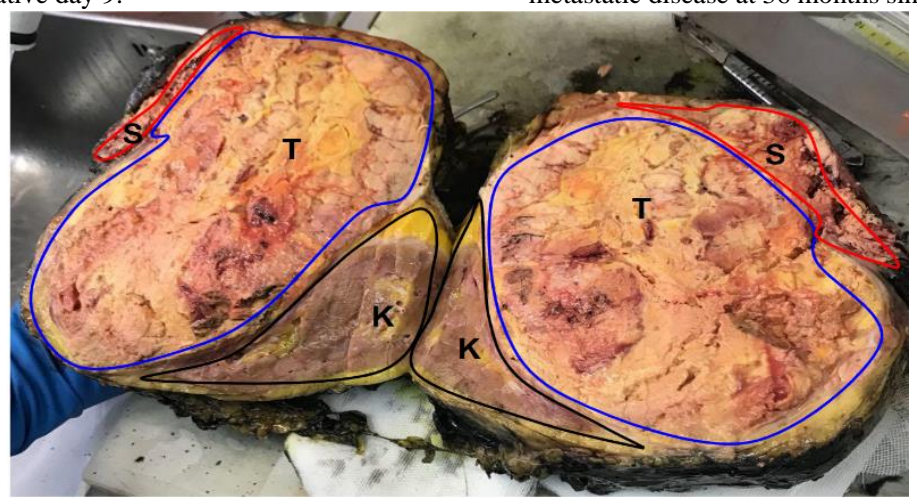

Figure 3: Macroscopic appearance of the resected specimen: giant yellow to brown mass, with some necrotic areas (K: Kidney; S: Spleen; T: Tumor).

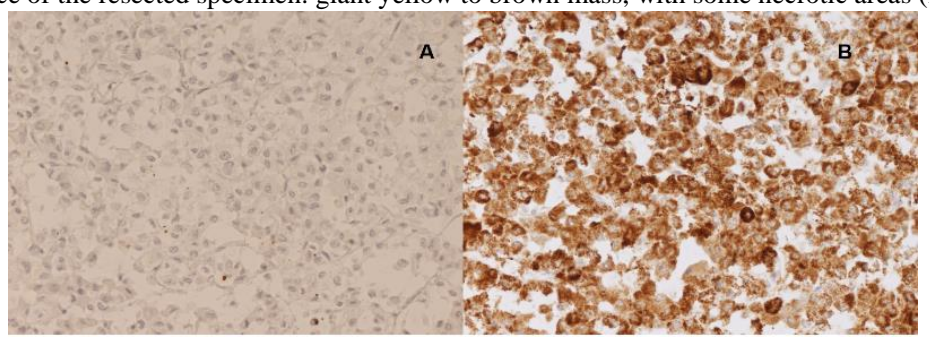

Figure 4: IHC showed A) CKAE1/AE3 negative and B) MelanA positive addressing for a definitive diagnosis of adreno cortical carcinoma.

\section{Discussion}

Surgical removal still remains the treatment of choice for ACC: whenever feasible, it is related with decreased cancer recurrence on tumoral site and increased overall survival rate; data from the MD Anderson Cancer Center, based on 311 treated patients over a 30-years period, demonstrated an $83.3 \%$ resectability rate (275/311), a clear prevalence for open procedures $(244 / 311 ; 88.7 \%)$, while an R0 resection has been achieved on $55.6 \%$ of patients (153/275). A large rate of resected patients received adjuvant treatment with mitotane alone or in combination with other chemotherapy $(235 / 331 ; 71.2 \%)$. Surgeon's experience, presence of R1/R2 resection margins and TNM Stage were significantly related to patient's prognosis at univariate analysis; factors such as gender, presence of hormonal dysfunction and side location were not related with local recurrence. Authors report an overall 5-year survival rate of $38 \%$, with a median survival time of 3.2 years since surgical treatment [7].

Extension of ACC to surrounding structures and absence of a clear cleavage at preoperative diagnostic work-up should not represent a contraindication to an aggressive surgical approach: several experiences dealing with giant ACCs treated with multivisceral removal have been reported with satisfactory results $[8,10,12-15]$. This surgical attitude has also been supported by larger consecutive series: data from the U.S. Adrenocortical Carcinoma Database show that 68 among 167 patients (40.7\%) underwent adrenalectomy with some en-bloc resection of additional organs (AdEBR) and 26 patients among them had multiple organs removed (mainly kidney, left liver lobe, spleen, pancreatic tail); in this subgroup larger tumor (13 vs. $10 \mathrm{~cm}$.), more open operation (97.1 vs. $63.6 \%$ ) and increased rate of lymph node dissection (36.8 vs. $12.1 \%$ ) 
have been observed when compared to the adrenalectomy alone subgroup (99 patients). Authors suggest current use of AdEBR whenever a T4 lesion is suspected or when appears mandatory to avoid tumor rupture. Size of the lesion, lymph node status, presence/absence of additional organ resection were not related to patient's overall survival at multivariate Cox regression (HR 1.42; $p=0.323$ ) [5]. Another consecutive series accounted for 35 patients with ACC operated over a 25-year period; 22/35 patients (63\%) underwent resection extended to spleen, pancreatic tail, inferior vena cava and left colon. Consistent rate of patients had postoperative adjuvant treatment with mitotane (17/35, $48.5 \%$ ). Authors report a 3- and 5-year overall survival rate of respectively $70.8 \%$ and $28.3 \%$, without significative differences between extended vs non-extended surgical resection; they strongly support aggressive approach even for locally advanced ACC as well as for treating local recurrences, independently from the primary stage of the disease [4].

Further improvement in early- and long-term results may be reached by preoperative angiography (with or without TAE) and adjuvant treatment with mitotane (alone or in combinations with other chemotherapy). Left large-sized ACC may supply blood from several arteries in left abdominal compartment, such as left adrenal artery, left renal artery through upper polar segment, splenic artery, pancreatic tail artery and middle colic artery [11]. This matter makes angiographic study an unavoidable step in diagnostic work-up: in-fact preoperative knowledge of arterial distribution in large sized ACC and possible presence of additional suppling vessels may be very useful for planning various aspects of surgical strategy (type of incision, medial-to-lateral vs. lateralto-medial reflection of surrounding structures). Accurate view of vessel distribution may be helpful in assessing adrenal origin of the mass as left hypochondrium may be occupied by a wide range of malignant pathologies: they include urologic lesions, such as renal cell carcinoma and transitional cell carcinoma of the renal pelvis; periaortic and retrocrural lymphadenopathies from testicular cancer or lymphoma; adrenal tumors, such as ACC and giant pheochromocytoma; primitive retroperitoneal malignancies; adenocarcinoma arising from distal transverse or splenic flexure of the colon as well as from pancreatic body and tail $[16,17]$.

Finally, preoperative TAE of tumor-supplying vessels by using low viscosity Precipitating Hydrophobic Injectable Liquid (Phil ${ }^{\circledR}$ ) 35\% agent may reduce intraoperative blood loss, avoiding or decreasing need for transfusions and reducing postoperative morbidity [10, 11]. Role for TAE has also been established in treating liver and/or spleen metastases from ACC as well as tumor bed recurrences $[3,11,18]$. In patient we treated, preoperative TAE and accurate surgical dissection significantly reduced intraoperative blood loss (estimated in $450 \mathrm{ml}$ ); this reduced amount, together with preoperative $\mathrm{Hb}$ level of $14.6 \mathrm{~g} / \mathrm{dl}$ led us to avoid transfusions. Intraoperative blood loss appears significantly higher whenever extended resection is not associated with preoperative TAE: in a consecutive series of 70 patients treated for extended urologic pathologies of left hypochondrium, mean estimated blood loss was 973 $\mathrm{ml}[16]$.

Once a definitive diagnosis of ACC has been established by immunohistochemical staining and additional information regarding prognosis are acquired through Weiss criteria and Ki67 index, some adjuvant treatment may be considered. Mitotane has been established as effective in the adjuvant treatment and in locally-advanced and metastatic ACC; however, direct antitumor effects have been demonstrated highly variable between patients in experimental studies [7, 19]. Moreover, results are strongly influenced by possible development of side effects (15-20\% of patients) and achievement of stable therapeutic blood level of $14-20 \mathrm{mg} / \mathrm{L}$ (30\% of patients) [2, 7]. In some series differences in overall survival rate have been observed between treated and non-treated patients, but they did not reach statistical significance [4]. At present adjuvant mitotane treatment is recommended in patients with high risk of disease recurrence (T4 lesion; Stage III disease; R1 resection; presence of Ki67> 10\%) but exact mitotane dose, side-effect profile and timing for starting therapy still need to be clarified [7]. Patients with widespread metastatic disease may benefit from systemic therapies, which combine mitotane with other agents, such as etoposide, doxorubicin, and cisplatin or, as an alternative, mitotane plus streptozotocin [2,7]. Potential benefits from adjuvant chemotherapy and radiotherapy in decreasing local recurrence and improving survival still need to be better clarified by prospective and multicenter studies $[7,20]$.

\section{Conclusion}

Combination of preoperative TAE, surgical aggressive approach with compartment removal and proper adjuvant treatment with mitotane may guarantee remarkable outcome and long-term disease-free survival rate, even in locally advanced ACC. Following this treatment strategy, we resected a giant left adrenal mass, performing an associated radical nephrectomy and distal splenopancreasectomy: resected specimen measured $24 \times 22 \times 12 \mathrm{~cm}$, weighted 2,080 gr. and probably represents one of the largest non-functioning adrenal mass ever operated. Length of follow up (36 months) and absence of tumor recurrence and/or distant metastases lead us to predict a favourable outcome.

\section{Conflicts of Interest}

None.

\section{Funding}

None.

\section{REFERENCES}

1. Favia G, Lumachi F, Carraro P, D'Amico DF (1995) Adrenocortical carcinoma. Our experience. Minerva Endocrinol 20: 95-99. [Crossref]

2. Fassnacht M, Dekkers O, Else T, Baudin E, Berruti A et al. (2018) European Society of Endocrinology Clinical Practice Guidelines on the management of adrenocortical carcinoma in adults, in collaboration with the European Network for the Study of Adrenal Tumors. Eur J Endocrinol 179: G1-G46. [Crossref]

3. Soga H, Takenaka A, Ooba T, Nakano Y, Miyake H et al. (2009) A twelve-year experience with adrenal cortical carcinoma in a single institution: long-term survival after surgical treatment and transcatheter arterial embolization. Urol Int 82: 222-226. [Crossref]

4. Borrelli D, Bergamini C, Borrelli A, Reddavide S, Lassig R et al. (2003) [Surgical strategy in the treatment of adrenal cortex cancer. Expanded and repeated interventions]. Ann Ital Chir 74: 311-317. [Crossref] 
5. Smith PM, Kiernan CM, Tran TB, Postlewait LM, Maithel SK et al. (2018) Role of Additional Organ Resection in Adrenocortical Carcinoma: Analysis of 167 Patients from the U.S. Adrenocortical Carcinoma Database. Ann Surg Oncol 25: 2308-2315. [Crossref]

6. Vaidya A, Nehs M, Kilbridge K (2019) Treatment of Adrenocortical Carcinoma. Surg Pathol Clin 12: 997-1006. [Crossref]

7. Ayala Ramirez M, Jasim S, Feng L, Ejaz S, Deniz F et al. (2013) Adrenocortical carcinoma: clinical outcomes and prognosis of 330 patients at a tertiary care center. Eur J Endocrinol 169: 891-899. [Crossref]

8. Bacalbasa N, Terzea D, Jianu V, Marcu M, Stoica C et al. (2015) Multiple visceral resection for giant non-secretory adrenocortical carcinoma in an elderly patient: a case report. Anticancer Res 35: 21692174. [Crossref]

9. Weiss LM (1984) Comparative histologic study of 43 metastasizing and nonmetastasizing adrenocortical tumors. Am J Surg Pathol 8: 163169. [Crossref]

10. Minato N, Yamaguchi Y, Koga M, Sugao H (2012) [Giant nonfunctioning adrenocortical carcinoma effectively treated with preoperative transarterial embolization: a case report]. Hinyokika Kiyo 58: 193-196. [Crossref]

11. Li SH, Huang CH, Ko SF, Chou FF, Huang SC (2005) Extended survival in a patient with recurrent and metastatic adrenal cortical carcinoma by aggressive transarterial embolization--a case report. $J$ Surg Oncol 90: 101-105. [Crossref]

12. Toledo GEM, Guerra JLL, Pérez AM, Marín IRP (2009) [Giant adrenal carcinoma. Case report]. Arch Esp Urol 62: 134-136. [Crossref]

13. Mantzoros I, Bitsianis S, Loutzidou L, Ntampakis G, Chatzakis C et al. (2021) Giant Adrenocortical Carcinoma: A Case Report and Review of the Relevant Literature. Am J Case Rep 22: e928875. [Crossref]
14. Chatzoulis G, Passos I, Bakaloudi DR, Giannakidis D, Koumpoulas A et al. (2018) Giant nonfunctioning adrenal tumors: two case reports and review of the literature. J Med Case Rep 12: 335. [Crossref]

15. Almarzouq A, Asfar S, Hussain S, Al Hunayan A, Aldousari S (2014) Giant nonfunctioning adrenocortical carcinoma: a case report and review of the literature. BMC Res Notes 7: 769. [Crossref]

16. Ciancio G, Vaidya A, Shirodkar S, Manoharan M, Hakky T et al. (2009) En bloc mobilization of the pancreas and spleen to facilitate resection of large tumors, primarily renal and adrenal, in the left upper quadrant of the abdomen: techniques derived from multivisceral transplantation. Eur Urol 55: 1106-1111. [Crossref]

17. Munakomi S, Rajbanshi S, Adhikary S (2016) Case Report: A giant but silent adrenal pheochromocytoma - a rare entity. F1000Res 5: 290. [Crossref]

18. Huszty G, Doros D, Farkas K, Kóbori L, Reismann P et al. (2021) Case Report: Complete Necrosis of a Large Adrenocortical Cancer and Liver Metastases Achieved by Selective Arterial Embolization: A Case Study and Review of Literature. Front Endocrinol (Lausanne) 12: 677187. [Crossref]

19. van Koetsveld PM, Creemers SG, Dogan F, Franssen GJH, de Herder WW et al. (2020) The Efficacy of Mitotane in Human Primary Adrenocortical Carcinoma Cultures. J Clin Endocrinol Metab 105: 407-417. [Crossref]

20. Gaujoux S, Weinandt M, Bonnet S, Reslinger V, Bertherat $\mathrm{J}$ et al. (2017) Surgical treatment of adrenal carcinoma J Visc Surg 154: 335343. [Crossref] 